\title{
Chronic Inflammatory Polyneuropathy Associated With Ulcerative Colitis: A Reported Case In Literature

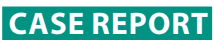

\section{Abstract}

Background: Peripheral neuropathy is known to be related to inflammatory bowel disease and it is one of the most frequently reported neurologic complications. Various studies have found peripheral nervous system complications, rather than central nervous system involvement, to be predominant. In the literature, there are a few cases of inflammatory polyneuropathy developed in the course of ulcerative colitis: 5 cases of Guillain-Barré syndrome, one case of chronic inflammatory demyelinating polyneuropathy, 10 cases of neuropathy and one case of perineuritis.

Case presentation: We describe a case of chronic inflammatory polyneuropathy associated with ulcerative colitis.

Conclusion: Peripheral neuropathy is not a common manifestation of inflammatory bowel disease, highlighting the need for careful exclusion of other causes of neuropathy when both conditions are encountered in clinical practice.

\section{Keywords}

Chronic inflammatory demyelinating polyneuropathy, Guillain-Barré syndrome, Nerve biopsy, Ulcerative colitis, inflammatory bowel disease

\section{Background}

Ulcerative colitis (UC) is a chronic relapsing inflammatory bowel disease (IBD) showing immunologic abnormalities and associated with autoimmune states. It affects individuals throughout their life. Extraintestinal manifestations of UC are well documented, but their association with neurological involvement is rare [1-8].

There are a few reports of neurologic involvement in this setting without previous medication. The occurrence of perineuritis associa-
Maria Sheila Guimarães

Rocha1,

Claude Vita| ${ }^{3,4}$

Alain Lagueny ${ }^{3,4}$, Luiz Carlos de Abreu², Alzira Alves de Siqueira Carvalho ${ }^{2}$

1 Neurology Department, Santa Marcelina Hospital, São Paulo, Brazil.

2 Neuroscience Department, ABC Medical School, Santo André, Brazil, and Study design Laboratory and Scientific Writing, Department of Community Health, ABC Medical School. Brazil.

3 Neurology Department, EA 2966 Neurobiology of Myelin Disorders.

4 Neurophysiology Department, CHU Bordeaux, Hôpital Pellegrin, France Bordeaux, France.

\section{Contact information:}

Alzira Alves de Siqueira Carvalho. Neuroscience Department, ABC Medical School, Santo André, Brazil and Study design Laboratory and Scientific Writing, Department of Community Health, $A B C$ Medical School. Brazil

झ alzira@myogenetica.com.br 
ted with UC has been reported in only one case by Chad et al. [2].

\section{Case presentation}

A 62-year-old caucasian woman was referred to the Hospital complaining of dorsal thoracic pain, muscular weakness and hypoesthesia of her legs for 4 months. In the last fifteen days she gets worsening of weakness and had become quadriparetic. Examination revealed a marked weakness of both legs and mild weakness of her arms (MRC grade 2 in lower limbs and grade 4 in upper limbs). Muscle tone was decreased and tendon reflexes were absent in the legs and decreased in her arms. Sensation of light touch was impaired in a "glove and stocking" type distribution in lower and upper limbs.

Vibration and position sensations were diminished in the legs. There was no sensory level to any modality. The cranial nerve functions were normal. The biochemical profile, liver functions, vitamin B12, folic acid, and thyroid hormones were normal. Serological tests for Epstein-Barr virus, cytomegalovirus, hepatitis $B$ and $C$ virus, and human immunodeficiency virus were all negative. The ESR was $75 \mathrm{~mm} /$ hour.

Collagen diseases and malignancy were ruled out by appropriate laboratory tests. Serum protein electrophoresis showed a mild increase of gammaglobulin. The CSF protein was $119 \mathrm{mg} / \mathrm{dl}$ (normal: 10-40 mg/dl). The myelogram and image studies (cervical and lombar MRI) were normal.

The conduction studies are shown in Table 1. Needle examination showed neurogenic patterns in both legs.

Neuromuscular biopsy revealed a inflammatory mononuclear cell infiltration in endoneurium with perivascular infiltrates without capillary wall modification and isolated inflammatory cells scattered also in endoneurium and moderate inflammatory infiltration of lymphocytes and monocytes in perivascular space also without inflammation in vessel walls of muscle (Fig. 1, 2, 3).

The patient was treated with IVG, 400 mg/kg/ day, five days, with no improvement of symptoms. Two weeks afterwards she developed intense rec-

Table 1. Conduction studies results of our patient.

\begin{tabular}{|l|c|c|c|c|c|}
\hline Nerves & MNCV $(\mathrm{m} / \mathrm{s})$ & CMAP amplitude $(\mathrm{mV})$ & DL $(\mathrm{ms})$ & SNVC $(\mathrm{m} / \mathrm{s})$ & SNAP amplitude $(\mu \mathrm{V})$ \\
\hline R median & 46.4 & 3.4 & 4.0 & ND & ND \\
\hline L median & 44.8 & 6.2 & 3.8 & 51. & 2.9 \\
\hline R ulnar & 53.0 & 7.6 & 3.4 & ND & 0 \\
\hline L ulnar & 50.0 & 8.6 & 2.9 & 48.2 & 0 \\
\hline R peroneal & 31.0 & 1.9 & 4.7 & & \\
\hline L peroneal & 29.1 & 0.8 & 5.6 & & \\
\hline R tibial & 34.9 & 4.6 & 5.3 & & \\
\hline L tibial & 37.0 & 4.0 & 4.1 & & 0 \\
\hline R sural & & & & ND & \\
\hline L sural & & & & ND & 0 \\
\hline
\end{tabular}

ND: not determinable; average(SD); R: right; L: left; Control values: MNCV, median= 55(4.5), DL= 3..8(0.4),

CMAP amplitude= 12(2.4); ulnar= 54,6(3.6), DL= 2,6(0,3), CMAP amplitude=9..5(2..5); peroneal= 49(5..5), DL=4..5(0.6),

CMAP amplitude =6.,3(1.6); tibial= 52.6, DL=3.0, CMAP amplitude= 24; $\operatorname{SNCV}$, median= 52(3.,5);

SNAP amplitude $=12.5(3.4)$; SNAP amplitude $=5.5$; sural=48(4), SNAP amplitude= 16(4..5). 
Figure 1. H\&E stain. Nerve: Perivascular lymphocyte inflammation in endoneurium.

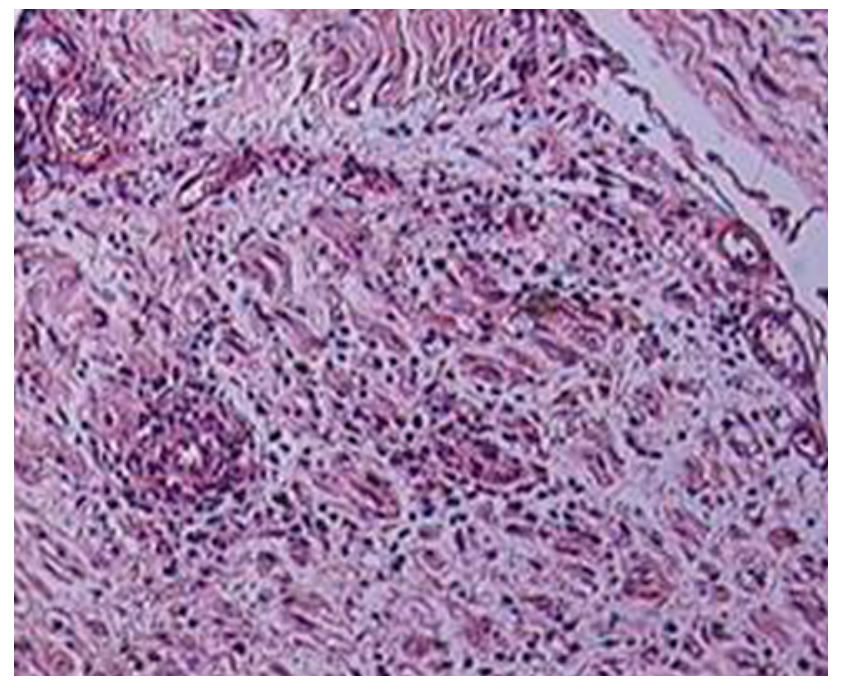

Figure 3. H\&E stain. Muscle: Perivascular lymphocyte inflammation in perimysium.

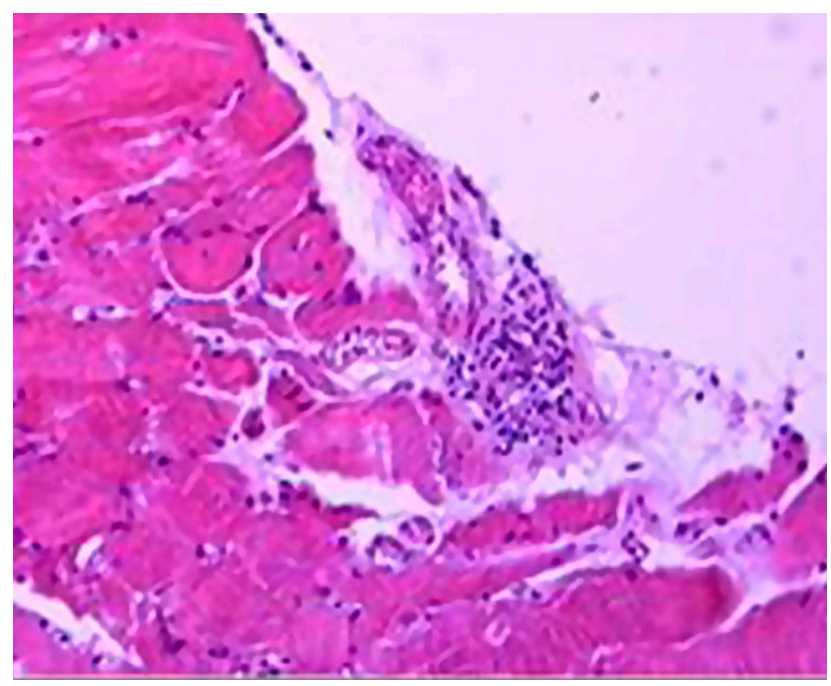

tal bleeding with feelings of weakness, dizziness, faintness and diarrhoea. The colonoscopy suggested UC and colon biopsy confirmed the diagnosis (Fig. 4). Therapy with steroid enemas and sulfasalazine, resulted in partial response. A sigmoidectomy was performed because the sigmoid colon was the most affected by colitis, which brought the patient to her normal control of intestinal symptoms. A pulse of methylprednisolone resulted in a pro-
Figure 2. H\&E stain. Nerve: Perivascular lymphocyte inflammation in perineurium.

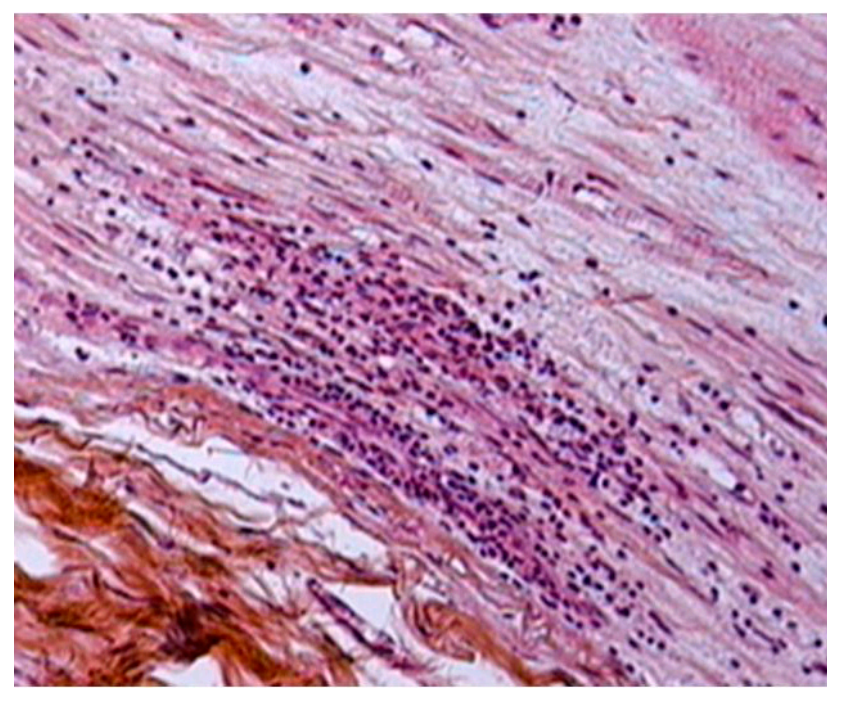

Figure 4. H\&E stain. Lymphocytic infiltration of the intestinal mucosa and architectural distortion of the crypts.

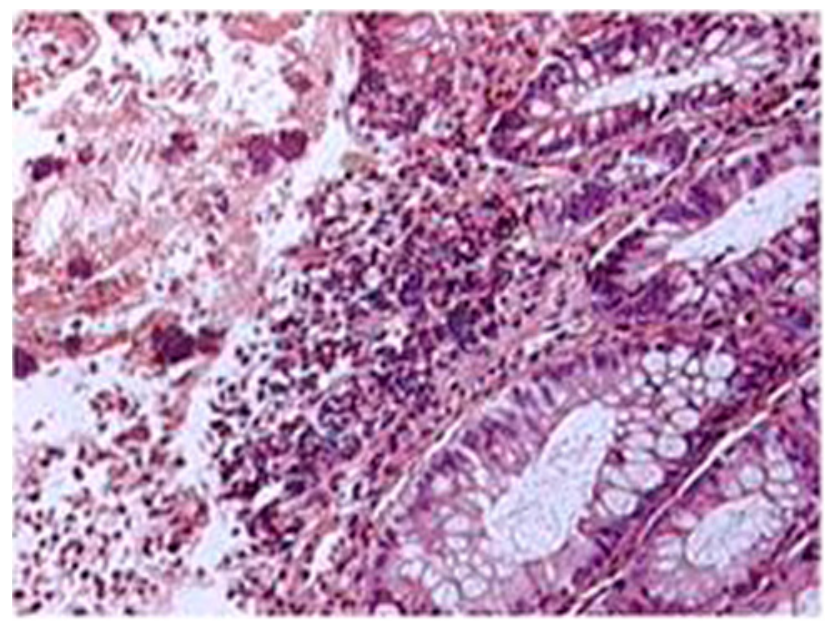

gressive improvement of neurological symptoms, making ambulation possible again. Azathioprine 2 $\mathrm{mg} / \mathrm{kg}$ daily was added and patient's neurological condition continued to improve for 3 years and after that the patient never returned. 


\section{Discussion}

Peripheral neuropathy (PN) is known to be related to IBD and it is one of the most frequently reported neurologic complications. Various studies have found peripheral nervous system complications, rather than central nervous system involvement, to be predominant. The reported frequency of PN in IBD will vary greatly among published studies, with estimates ranging from $0 \%$ to $39 \%$ due to selection bias, use of different definitions of the disease, or population characteristics [9].

Ulcerative colitis (UC) is a chronic relapsing inflammatory bowel disease with various extra-intestinal manifestations and there are a few reports of neurologic involvement in this setting without previous medication (Table 2). In 1985, 2 elderly patients with Guillain-Barré syndrome (GBS) were reported while the UC was in remission [1]. In 1989, a case of chronic polyneuropathy was reported associated with UC [3]. In the same year, in a series of 60 cases of CIDP, Barohn et al briefly mentioned one patient who also had inflammatory bowel disease [10].

Lossos et al. reported a patient (case 3) with acute demyelinating polyradiculoneuropathy 12 years after the UC had started [4]. A case of GBS was described in a patient with the diagnosis of UC 30 years earlier [5]. A congenital GBS associated with maternal UC was reported by Bamford et al. [11]. A 6-year-old girl developed an axonal sensorimotor polyneuropathy a few days before intestinal symptoms with bloody diarrhea and abdominal pain. Colon biopsy established the diagnosis of ulcerative colitis [6]. In 2005, Gondin et al presented the largest case series of PN in patients with IBD. Both demyelinating (30\%) and non-demyelinating neuropathies were observed. Eighteen patients with $C D$ and 15 patients with UC were identified after other PN causes were excluded [12].

A case reported by Yesilova et al described an axonal motor polyneuropathy in a patient with ulcerative colitis. Symptoms of neuropathy occurred during active colitis [8].

In 2007, Zezos et al reported a case of acute motor and sensory neuropathy during a flare of ulcerative colitis in a 28-year-old male despite treatment with mesalazine enemas and suppositories simultaneously with rapidly deteriorating weakness and needle sensation in both legs [7].

In 2010, a 59-year-old male patient developed GBS during relapse of UC [13].

Table 2. Characteristics of 30 patients with UC who developed peripheral neuropathy.

\begin{tabular}{|l|c|c|c|c|c|}
\hline Author & year & gender & age & Num cases & type \\
\hline Zimmerman et al & 1985 & $\mathrm{M}$ & 59 & 2 & GBS \\
\hline Chad et al & 1986 & $\mathrm{M}$ & 50 & 2 & $\mathrm{PN}$ \\
\hline Konayaga et al & 1989 & $\mathrm{M}$ & 57 & 1 & $\mathrm{PN}$ \\
\hline Roca et al & 1999 & $\mathrm{~F}$ & 69 & 1 & $\mathrm{GBS}$ \\
\hline Bamford et al & 2002 & $\mathrm{NO}$ & $34 \mathrm{~W}$ & 1 & $\mathrm{GBS}$ \\
\hline Greco et al & 2004 & $\mathrm{~F}$ & 6 & 1 & $\mathrm{PN}$ \\
\hline Gondin et al & 2005 & $11 \mathrm{M} / 4 \mathrm{~F}$ & $24-72$ & 15 & $\mathrm{PN}$ \\
\hline Yesilova et al & 2006 & $\mathrm{M}$ & 47 & 1 & $\mathrm{PN}$ \\
\hline Zezos et al & 2007 & $\mathrm{M}$ & 28 & 1 & $\mathrm{PN}$ \\
\hline Kristallis et al & 2010 & $\mathrm{M}$ & 59 & 1 & GBS \\
\hline Figueroa et al & 2013 & 3M/1F & $51-85$ & 4 & RPN/PN \\
\hline PN: peripheral neuropathy, GBS: Guillain-Barré syndrome, RPN: radiculoplexus neuropathy, NO:not obtained
\end{tabular}


A population-based cohort study demonstrated 772 patients diagnosed with IBD(UC and Crohn disease(CD)). Only nine patients, aged 34 to 86 years developed peripheral neuropathy. Six out 9, with UC and 3 with CD. The neuropathies comprised two phenotypes: radiculoneuropathy and chronic sensorimotor distal polyneuropathy. However, 3 patients were diabetic [14]. The clinical and electrophysiologic features of our patient were compatible with axonal polyneuropathy with MNCV abnormalities suggesting additional demyelinating features.

The occurrence of perineuritis associated with UC has been reported in only one case by Chad et al. [2]. Their patient had a chronic polyneuropathy and the CSF protein content was $875 \mathrm{mg} /$ dl. He showed a gradual improvement with steroid therapy.

King emphasized that the cellular infiltration is more conspicuous in the epineurium in vasculitis whereas there is an infiltration in the endoneurium in autoimmune diseases [15].

Our patient showed only cellular infiltration in endoneurium and the diseases most commonly associated with vasculitis in endoneurium were excluded. All the cases cited above did not mentioned about nerve biopsy. Also, they did not specified the location of involvement by inflammatory cells except the paper published by Chad et al [2].

The pathogenesis of UC remains unclear but evidence suggests that an auto-immune antibodymediated mechanism is involved.

\section{Conclusion}

Taken together, the data suggest that the neuropathy reported in our patient is inflammatory probably due to an autoimmune-mediated mechanism suggesting that both diseases had a common pathogenesis.

The findings reported above show that PN in IBD is not a common manifestation of IBD, highlighting the need for careful exclusion of other causes of neuropathy when both conditions are encountered in clinical practice.

\section{Consent}

Written informed consent was obtained from the patient for publication of this case report and any accompanying images. A copy of the written consent is available for review by the Editor of this journal.

\section{Competing interests}

The authors declare that they have no competing interests.

\section{Authors' contributions}

All authors participated in results collection, study design and manuscript draft. All authors agreed with the final version of the manuscript.

\section{Acknowledgements}

The authors state that the study received financial support from Foundation of Support to Research of Sao Paulo State (FAPESP) and Neuroscience Department of ABC Medical School. 


\section{References}

1. Zimmerman J, Steiner I, Gavish D, Argov Z. Guillain-Barré Syndrome: a possible extraintestinal manifestation of ulcerative colitis. J Clin Gastroenterol 1985, 7: 301-303.

2. Chad DA, Smith TW, DeGirolami U, Hammer K. Perineuritis and ulcerative colitis. Neurology 1986, 36: 1377-1379.

3. Konagaya Y, Konagaya M, Takayanagi T. Chronic polyneuropathy and ulcerative colitis. Jpn J Med 1989, 28: 72-74.

4. Lossos A, River Y, Eliakim A, Steiner I. Neurologic aspects of inflammatory bowel disease. Neurology 1985, 45: 416-421.

5. Roca B, Moreno I, Meneu E. Ulcerative colitis and acquired demyelinating neuropathy (Guillain-Barré syndrome). Neth J Med 1999, 54: 129-130.

6. Greco F, Pavone P, Falsaperla R, Sorge G.J. Peripheral neuropathy as first sign of ulcerative colitis in a child. Clin Gastroenterol. 2004, Feb; 38(2): 115-7.

7. Yeşilova Z, Naharci I, Uygun A, Ulaş HU, Dağalp K. Motor axonal polyneuropathy in the course of ulcerative colitis: a case report. Turk J Gastroenterol. 2006 Mar; 17(1): 58-61.

8. Zezos P, Mpoumponaris A, Koutsopetras P, Vounotrypidis P, Molyvas E, Vadikolias K, Moschos I, Kouklakis. Acute motor sensory polyneuropathy (AMSAN) complicating active ulcerative colitis with a patchy distribution. G.Acta Gastroenterol Belg. 2007 Apr-Jun; 70(2): 226-30.

9. Moris G. Inflammatory bowel disease: an increased risk factor for neurologic complications.World I Gastroenterol 2014, Feb 7; 20(5): 1228-37.

10. Barohn RJ, Kissel JT, Warmolts JR, Mendell JR. Chronic inflammatory demyelinating polyradiculoneuropathy : clinical characteristics, course, and recommendations for diagnostic criteria. Arch Neurol 1989, 46: 878-884.

11. King King RHM. Atlas of Peripheral Nerve Pathology. London: Arnold; 1999: 1 \pm 217

12. F. A. A. Gondim, T. H. Brannagan, H. W. Sander, R. L. Chin, N. Latov. Peripheral neuropathy in patients with inflammatory bowel disease. Brain. 2005 Apr; 128 (Pt 4): 867-79.

13. Krystallis CS, Kamberoglou DK, Cheilakos GB, Maltezou MN, Tzias VD. Guillain-Barré syndrome during a relapse of ulcerative colitis: a case report. Inflamm Bowel Dis 2010 Apr; 16(4): 555-6.

14. Figueroa JJ, Loftus EV Jr, Harmsen WS, Dyck PJ, Klein CJ. Peripheral neuropathy incidence in inflammatory bowel disease: a population-based study. Neurology. 2013, Apr 30; 80(18): 1693-7.

15. King RHM. Atlas of peripheral nerve pathology, 1999 Arnold, London, pp 1-217.

\section{Comment on this article:}

\section{(f) (B) in $8+\boldsymbol{S}$ ?}

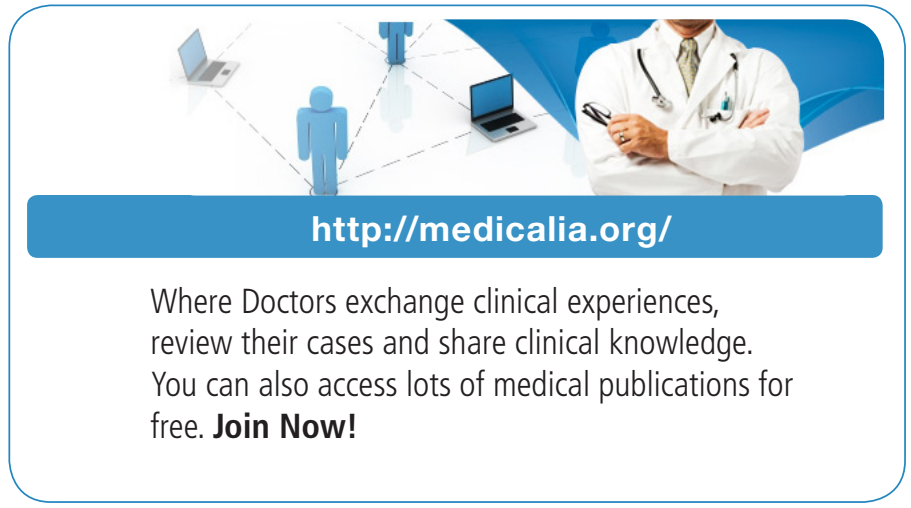

\section{Publish with iMedPub}

\section{http://www.imed.pub}

International Archives of Medicine is an open access journal publishing articles encompassing all aspects of medical science and clinical practice. IAM is considered a megajournal with independent sections on all areas of medicine. IAM is a really international journal with authors and board members from all around the world. The journal is widely indexed and classified Q1 in category Medicine. 